\title{
19. DATA REPORT: CLAST AND GRAIN-SIZE ANALYSIS OF SEDIMENT RECOVERED FROM THE NAPOLI AND MILANO MUD VOLCANOES, EASTERN MEDITERRANEAN ${ }^{1}$
}

\author{
Rachel Flecker ${ }^{2}$ and Achim Kopf ${ }^{3}$
}

\begin{abstract}
Comprehensive two-dimensional measurement of the clasts recovered from Sites 970 and 971 was conducted on board JOIDES Resolution, but could be presented only in a simplified form in the time available. These extensive databases were therefore processed post-cruise. Furthermore, as a result of a malfunction of the shipboard grain-size analyzer it was not possible to obtain grain-size information during Leg 160. Therefore, particle-size analysis on mud volcano samples was conducted post-cruise. The results of these initial studies are presented in this data report and form the basis for further post-cruise research.
\end{abstract}

\section{INTRODUCTION}

Two transects were drilled across the Milano (Site 970; "Geological Setting" section and Fig. 6, "Site 970" chapter, this volume) and Napoli (Site 971; "Geological Setting" section and Fig. 11, "Site 971 " chapter, this volume) mud volcanoes in the Olimpi mud diapir field on the Mediterranean Ridge. Sediment recovered from these sites is dominantly mud breccia of different types, interfingered with sapropel-bearing nannofossil ooze ("Lithostratigraphy" section, "Site 970" and "Site 971" chapters, this volume). The mud breccias contain variable quantities of clasts, which are dominated by muds, mudstones, and claystones (Tables 1,2 [CD-ROM, back pocket]). At both sites, these sediments are believed to be deposited by debrisflow processes (cf., "Summary and Conclusions" section, "Site 970" and "Site 971 " chapters, this volume).

\section{GRAIN-SIZE ANALYSIS}

\section{Sampling}

Eighty-two representative samples of mud breccia matrix were collected in order to determine grain-size variations among the holes and with depth. Clast-rich sequences such as the intervals of polymictic gravel (e.g., Cores 160-970A-18X through 22X) were not sampled.

\section{Method}

Grain-size analysis was conducted using a Coulter LS 100 particle-size analyzer, which uses the variation of an electrical current across an aperture to determine the volume of individual suspended sediment particles (cf., McManus, 1988). Seventy-four intervals between 0.4 and $900 \mu \mathrm{m}$ of variable magnitude (i.e., 0.045 to $91.5 \mu \mathrm{m}$ ) were then summed into grain-size classes of clay, silt, and sand as outlined in Tucker (1981).

Samples weighing between 0.21 and $0.78 \mathrm{~g}$ were placed in 100 $\mathrm{mL}$ of $4 \mathrm{~mol} \%$ sodium hexametaphosphate ("Calgon") solution for $24 \mathrm{hr}$ to deflocculate. The dispersed samples were then sieved to

'Emeis, K.-C., Robertson, A.H.F., Richter, C., et al., 1996. Proc. ODP, Init. Repts., 160: College Station, TX (Ocean Drilling Program).

2Department of Geology and Geophysics, Edinburgh University, West Mains Road, Edinburgh EH9 3JW, United Kingdom. flecker@ castle.ed.ac.uk

${ }^{3}$ Institut für Geowissenschaften und Lithosphärenforschung, Universität Giessen, Senckenbergstrasse 3,35390 Giessen, Germany. eliminate the fraction coarser than $500 \mu \mathrm{m}$. This coarse-grained component, which was found in the majority of samples, was weighed after desiccation (at $60^{\circ} \mathrm{C}$ overnight in an oven) and added to the sand component derived from the Coulter counter analysis. The possible error resulting from water loss can be neglected as all the samples had been partially air dried in the sample bags.

\section{Data}

The summarized results of the grain-size analysis are presented as stacked histograms. None of the samples analyzed contained particles coarser than sand grade (Figs. 1,2). Tables 3 and 4 also contain these data together with some statistical parameters. Standard deviation as a measure of sorting and the grain size itself are plotted against mean grain size in Figure 3. All samples have particle-size distributions that are skewed to the right of a Gaussian frequency distribution curve.

\section{CLAST-SIZE ANALYSIS \\ Clast-area Determination}

The number of clasts measured in any section depended on the abundance of clasts in the mud breccias. Clast frequency was highly variable, but no more than 50 clast measurements were made on any section. Only the largest clasts exposed on the core face were measured, so that the mean clasts size recorded in Tables 1 and 2 (CDROM, back pocket) is an average of the population of the largest clasts per section.

Volumetric clast data could not be obtained because of the difficulty of removing clasts from a matrix of similar lithification. The clast data are therefore presented here as the product of the visually estimated long and short axes of the area exposed on the core face. Calculating this product (i.e., a rectangle) results in an overestimation of the area that is at a maximum for perfectly rounded and triangular clasts. The systematic error that results is neglected in this purely comparative study on the grounds that the majority of the clasts are subangular to subrounded in shape.

\section{Data}

The clast data collected during Leg 160 shows that clast frequency and distribution vary significantly. The mean clast area presented on a hole-by-hole basis for the Milano and Napoli Domes (Figs. 1 and 2, respectively) reflects these variations. The data bases (Tables 1, 2 

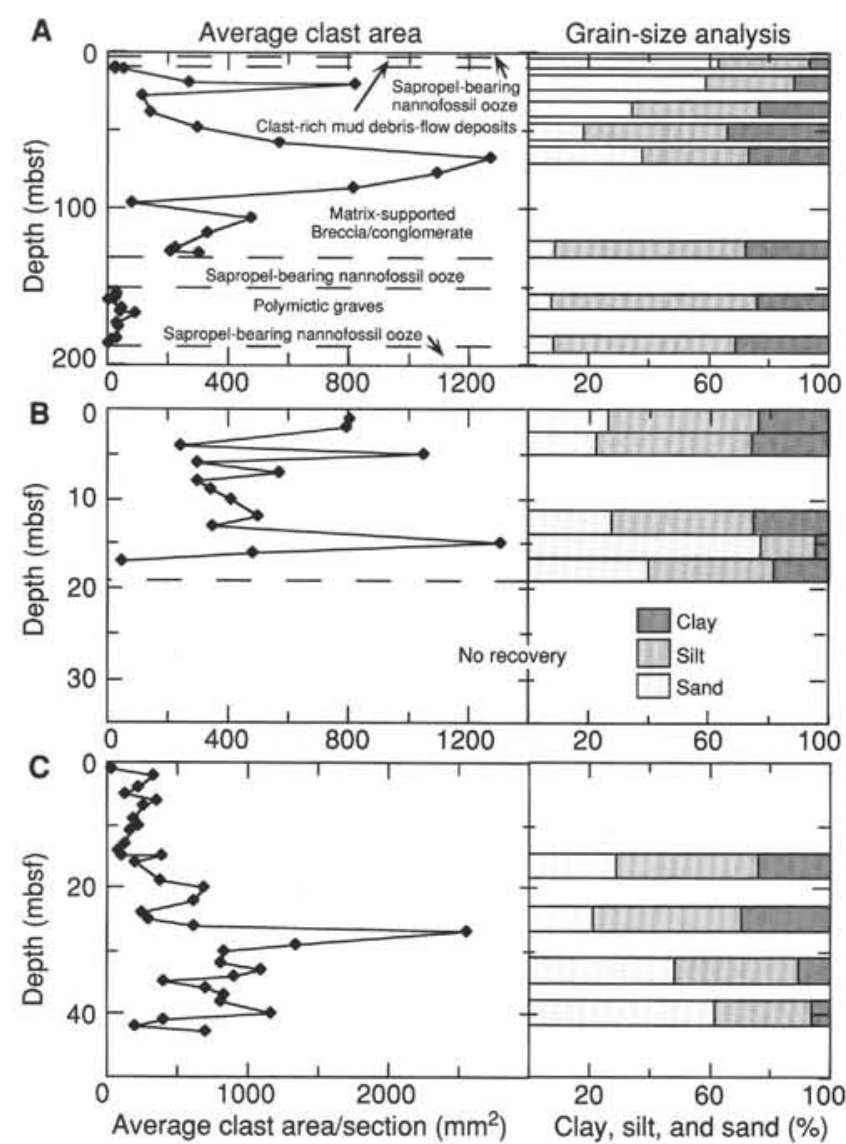

Figure 1. Average clast area per section and grain-size distribution vs. depth of mud volcano deposits from (A) Hole 970A, (B) Hole 970C, and (C) Hole 970D.

[CD-ROM, back pocket]) include information on the area, shape, and composition for each clast measured, with clast:matrix ratio, texture (matrix or clast supported), and average clast area determined per section.

\section{SUMMARY OF RESULTS}

A few comparisons can be made of the grain-size and clast-area data in terms of the different location of holes and sites. These can be summarized as follows:

1. The average clast area is considerably larger in the cores collected at the Milano Dome (Site 970) than at the Napoli mud volcano (Site 971; Figs. 1, 2).

2. The percentage of material coarser than $62 \mu \mathrm{m}$ in the matrix (i.e., sand component) is greater in the Milano Dome samples than in the ones from Napoli Dome (e.g., Milano Dome generally $>20 \%$ sand; Tables, 3, 4, Figs. 1, 2).

3. The flank and crest holes at the Milano mud volcano (i.e., Holes 970C and 970D) are rich in sand relative to the holes at the base of the slope (Hole 970A; cf., Fig. 1). These data indicate that the earlier mud breccias consisted of distinctly finer grained material than later ones.

4. The crest holes at the Napoli Dome (i.e., Holes 971D and 971E) contain very little sand and are, in general, significantly
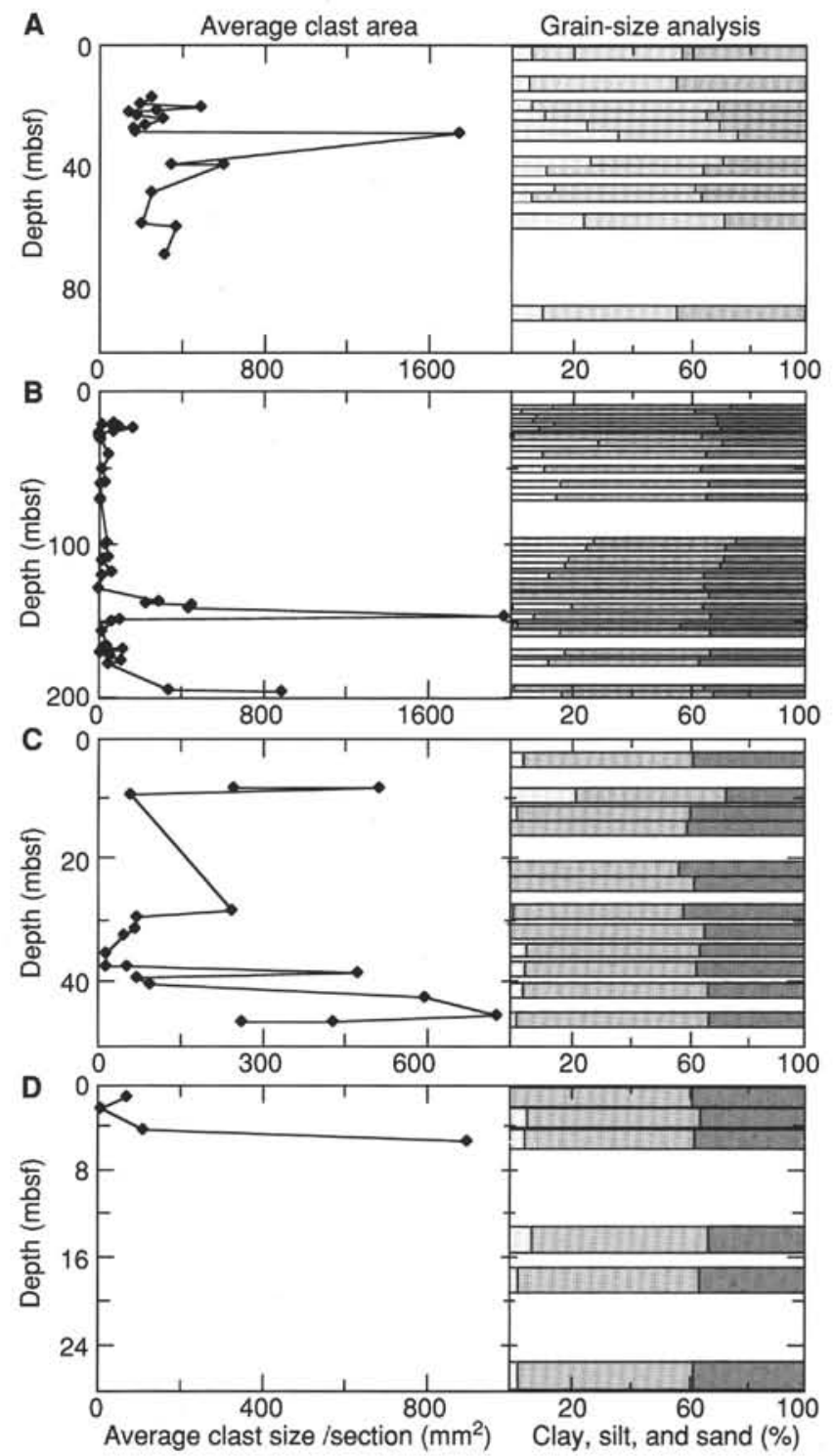

Figure 2. Average clast area per section and grain-size distribution vs. depth of mud volcano deposits from (A) Hole 971A, (B) Hole 971B, (C) Hole 971D, and (D) Hole 971E. Patterns as in Figure 1.

finer grained than sediment recovered at sites located in (Hole 971B) and beyond (Hole 971A) the moatlike feature at the base of the volcano structure.

5. The trend of the standard deviation data plotted against mean grain size suggests that sorting is related to grain size such that, in general, the coarser the grain size the poorer the sorting (Fig. 3).

6. Standard deviation for all samples ranges from 0.81 to 3.29 and indicates moderate to very poor sorting (Folk, 1974; cf., Tables 1, 2 [CD-ROM, back pocket]).

7. Bearing in mind the considerable errors associated with the comparison of two- and three-dimensional data, the occurrence and abundance of sand in several of the holes show a correspondence with the variation of mean clast size in plots against depth (e.g., Holes 970C and 971D; Figs. 1B and 2C, respectively). Interpretation of this relationship is not possible 
without further information (e.g., source of clast and matrix material, energy regime and dynamics of deposition, age, diagenesis of sediment).

\section{ACKNOWLEDGMENTS}

We would like to thank Angelo Camerlenghi and Mitch Malone for their helpful reviews and detailed suggestions. Impartial encouragement was exuded by Alastair Robertson. Anne Mennim is acknowledged for supporting the particle-size analyses. Astrid Kopf and Mejse Dairies provided generous support to the authors whilst writing the manuscript. Post-cruise financial support was provided by NERC and DFG.

\section{REFERENCES}

Folk, R.L., 1974. Petrology of Sedimentary Rocks: Austin, TX (Hemphill). McManus, J., 1988. Grain size determination and interpretation. In Tucker, M. (Ed.), Techniques in Sedimentology: Oxford (Blackwell), 63-85.

Tucker, M.E., 1981. An Introduction to Sedimentary Petrology: Oxford (Blackwell).

Ms 160IR-117

Table 3. Results of grain-size analyses from Site 970, Milano Dome.

\begin{tabular}{|c|c|c|c|c|c|c|c|c|}
\hline $\begin{array}{l}\text { Core, section, } \\
\text { interval }(\mathrm{cm})\end{array}$ & $\begin{array}{l}\text { Depth } \\
\text { (mbsf) }\end{array}$ & $\begin{array}{l}\text { Clay } \\
(\%)\end{array}$ & $\begin{array}{l}\text { Silt } \\
(\%)\end{array}$ & $\begin{array}{l}\text { Sand } \\
(\%)\end{array}$ & $\begin{array}{l}\text { Standard } \\
\text { deviation }\end{array}$ & Kurtosis & Mean & Skewness \\
\hline \multicolumn{9}{|l|}{ 160-970A- } \\
\hline IH-4, 65-67 & 5.15 & 6.42 & 30.50 & 63.63 & 0.90 & Platykurtic & 125.70 & 0.79 \\
\hline $4 X-1,44-46$ & 18.64 & 11.87 & 29.17 & 58.96 & 1.27 & Leptokurtic & 15.46 & 4.03 \\
\hline $6 X-1,14-17$ & 37.54 & 23.39 & 41.99 & 34.62 & 1.67 & Leptokurtic & 19.52 & 3.22 \\
\hline $7 X-1,19-22$ & 47.29 & 33.71 & 48.11 & 18.18 & 0.99 & Leptokurtic & 8.62 & 1.50 \\
\hline $9 X-1,62-65$ & 66.92 & 26.49 & 35.50 & 38.01 & 1.75 & Leptokurtic & 41.19 & 2.47 \\
\hline $11 \mathrm{X}-\mathrm{CC}, 9-10$ & 85.79 & 36.78 & 54.26 & 8.96 & 1.23 & Leptokurtic & 11.71 & 2.17 \\
\hline $15 X-1,110-112$ & 125.50 & 27.51 & 63.53 & 8.96 & 0.97 & Leptokurtic & 12.92 & 1.41 \\
\hline $18 X-2,92-94$ & 155.42 & 24.06 & 68.36 & 7.58 & 0.89 & Leptokurtic & 10.81 & 1.89 \\
\hline $18 X-3,29-31$ & 156.29 & 35.03 & 61.89 & 3.08 & 0.88 & Leptokurtic & 8.73 & 1.32 \\
\hline $18 X-3,68-70$ & 156.68 & 27.60 & 61.97 & 10.43 & 1.56 & Leptokurtic & 24.08 & 2.95 \\
\hline $22 \mathrm{X}-1,147-149$ & 193.17 & 30.89 & 60.84 & 8.27 & 1.41 & Leptokurtic & 16.07 & 2.56 \\
\hline \multicolumn{9}{|l|}{$160-970 \mathrm{C}$} \\
\hline IH-1, 83-84 & 0.83 & 23.58 & 50.01 & 26.42 & 2.11 & Leptokurtic & 74.86 & 2.82 \\
\hline $1 \mathrm{H}-2,1-3$ & 1.51 & 25.45 & 51.93 & 22.62 & 2.40 & Leptokurtic & 50.39 & 3,95 \\
\hline $3 \mathrm{H}-3,140-142$ & 12.60 & 24.74 & 47.58 & 27.68 & 1.42 & Leptokurtic & 14.69 & 3.14 \\
\hline $3 \mathrm{H}-5, \mathrm{I}-3$ & 14.21 & 26.44 & 51.76 & 21.80 & 2.12 & Leptokurtic & 41.66 & 3.44 \\
\hline $3 \mathrm{H}-5,73-75$ & 14.93 & 4.49 & 18.33 & 77.18 & 1.34 & Leptokurtic & 16.37 & 3.88 \\
\hline $4 X-1.9-12$ & 16.59 & 18.32 & 41.40 & 40.28 & 1.45 & Leptokurtic & 91.15 & 1.99 \\
\hline \multicolumn{9}{|l|}{$160-970 \mathrm{D}-$} \\
\hline $3 \mathrm{H}-1,47-54$ & 14.77 & 23.81 & 47.27 & 28.92 & 2.17 & Leptokurtic & 52.49 & 3.48 \\
\hline $3 \mathrm{H}-6,42-49$ & 22.22 & 29.18 & 49.83 & 20.99 & 2.52 & Leptokurtic & 62.34 & 3.30 \\
\hline $4 \mathrm{H}-5,52-54$ & 29.93 & 10.34 & 41.51 & 48.15 & 0.81 & Platykurtic & 16.76 & 0.84 \\
\hline $5 \mathrm{H}-2,61-64$ & 35.34 & 6.13 & 32.18 & 61.69 & 1.01 & Platykurtic & 191.50 & 0.90 \\
\hline
\end{tabular}

Note: Statistical parameters (standard deviation, kurtosis, skewness) defined in McManus (1988).

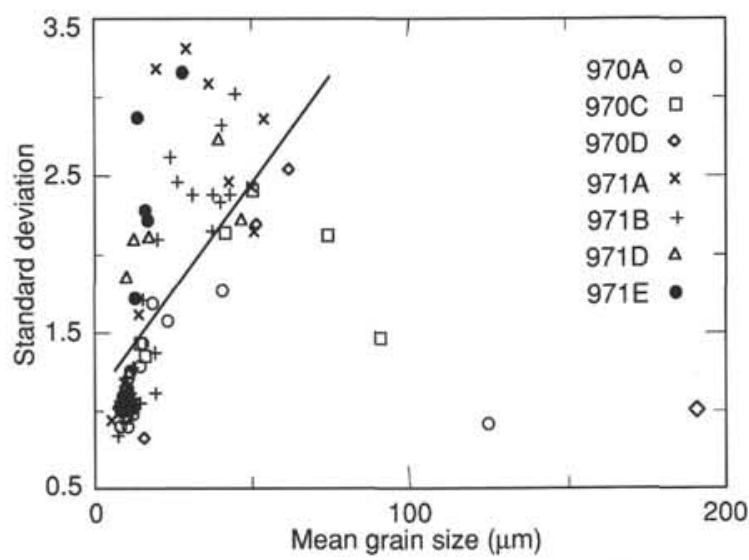

Figure 3. Standard deviation vs, mean grain size for Holes 970A, 970C, 970D, 971 A, 971B, 971D, and 971E. A best-fit line $(y=0.0274 x+1.0781, r$ $=0.6467)$ is shown for the data. 
Table 4. Results of grain-size analyses from Site 971, Napoli Dome.

\begin{tabular}{|c|c|c|c|c|c|c|c|c|}
\hline $\begin{array}{l}\text { Core, section, } \\
\text { interval }(\mathrm{cm})\end{array}$ & $\begin{array}{l}\text { Depth } \\
\text { (mbsf) }\end{array}$ & $\begin{array}{l}\text { Clay } \\
(\%)\end{array}$ & $\begin{array}{l}\text { Silt } \\
(\%)\end{array}$ & $\begin{array}{l}\text { Sand } \\
(\%)\end{array}$ & $\begin{array}{l}\text { Standard } \\
\text { deviation }\end{array}$ & Kurtosis & Mean & Skewness \\
\hline \multicolumn{9}{|l|}{ 160-971A- } \\
\hline IH-1, 52-54 & 0.52 & 41.96 & 51.25 & 6.79 & 3.29 & Leptokurtic & 29.61 & 5.34 \\
\hline $2 \mathrm{H}-5,50-52$ & 14.00 & 46.79 & 53.21 & 6.10 & 0.93 & Leptokurtic & 6.39 & 1.80 \\
\hline $3 \mathrm{H}-1,25-27$ & 16.75 & 32.98 & 67.02 & 0.00 & 0.99 & Leptokurtic & 10.21 & 1.86 \\
\hline $4 \mathrm{H}-1,100-102$ & 23.50 & 30.22 & 63.10 & 6.69 & 1.04 & Leptokurtic & 12.23 & 1.73 \\
\hline $4 \mathrm{H}-3, \mathrm{I} 00-102$ & 26.50 & 33.67 & 55.49 & 10.85 & 1.15 & Leptokurtic & 11.35 & 2.03 \\
\hline $5 X-C C, 2-4$ & 28.52 & 23.33 & 40.81 & 35.86 & 1.59 & Leptokurtic & 14.65 & 5.37 \\
\hline $6 \times-1,10-12$ & 38.30 & 28.08 & 45.10 & 26.82 & 2.13 & Leptokurtic & 51.25 & 3.16 \\
\hline $6 \mathrm{X}-\mathrm{CC}, 13-16$ & 39.04 & 35.16 & 53.04 & 11.80 & 3.07 & Leptokurtic & 37.42 & 4.89 \\
\hline $7 X-1.2-5$ & 47.92 & 37.75 & 47.73 & 14.52 & 1.17 & Leptokurtic & 10.26 & 1.69 \\
\hline $7 X-1,59-61$ & 48.70 & 35.35 & 57.87 & 6.78 & 1.02 & Leptokurtic & 9.76 & 1.63 \\
\hline $8 X-1,5-7$ & 57.65 & 27.54 & 47.80 & 24.66 & 2.44 & Leptokurtic & 43.84 & 4.15 \\
\hline $8 X-C C, 5-7$ & 58.07 & 29.57 & 44.91 & 25.52 & 1.05 & Leptokurtic & 10.05 & 1.54 \\
\hline $9 \mathrm{X}-\mathrm{CC}, 7-9$ & 67.37 & 38.61 & 55.36 & 6.03 & 3.17 & Leptokurtic & 20.13 & 7.28 \\
\hline $11 X-1,138-140$ & 87.50 & 43.06 & 45.07 & 10.67 & 2.84 & Leptokurtic & 54.47 & 3.58 \\
\hline $11 X-5,136-138$ & 95.70 & 33.29 & 50.49 & 14.90 & 2.41 & Leptokurtic & 50.28 & 3.79 \\
\hline \multicolumn{9}{|l|}{ 160-97IB- } \\
\hline $3 \mathrm{H}-5,117-119$ & 18.87 & 29.37 & 69.78 & 16.37 & 1.05 & Leptokurtic & 14.91 & 1.60 \\
\hline $3 \mathrm{H}-6,121-123$ & 20.41 & 37.55 & 59.11 & 3.33 & 1.11 & Leptokurtic & 10.29 & 1.92 \\
\hline $3 \mathrm{H}-7,26-28$ & 20.96 & 29.04 & 61.73 & 9.23 & 0.98 & Leptokurtic & 11.35 & 1.63 \\
\hline $4 \mathrm{H}-1,111-113$ & 22.31 & 29.75 & 56.05 & 14.20 & 2.62 & Leptokurtic & 24.40 & 5.80 \\
\hline $4 \mathrm{H}-2,12-14$ & 22.82 & 29.81 & 62.94 & 7.25 & 0.99 & Leptokurtic & 11.40 & 1.68 \\
\hline $4 \mathrm{H}-3,126-128$ & 25.46 & 30.52 & 61.25 & 8.23 & 2.10 & Leptokurtic & 20.52 & 5.42 \\
\hline $4 \mathrm{H}-6,111-113$ & 29.81 & 35.09 & 62.92 & 1.39 & 1.11 & Leptokurtic & 11.61 & 1.95 \\
\hline $6 \mathrm{X}-1,44-46$ & 40.14 & 28.34 & 42.23 & 29.43 & 1.71 & Leptokurtic & 15.59 & 3.83 \\
\hline $7 X-1,36-38$ & 49.66 & 33.62 & 55.98 & 10.40 & 1.27 & Leptokurtic & 12.26 & 2.70 \\
\hline $8 \mathrm{X}-\mathrm{CC}, 22-24$ & 59.93 & 35.30 & 53.81 & 10.89 & 2.81 & Leptokurtic & 40.20 & 4.52 \\
\hline $9 X-1,102-104$ & 69.62 & 32.56 & 50.78 & 16.65 & 2.38 & Leptokurtic & 37.93 & 3.91 \\
\hline $12 X-1,108-110$ & 98.58 & 23.49 & 48.87 & 28.25 & 1.02 & Leptokurtic & 11.70 & 1.70 \\
\hline $12 \mathrm{X}-\mathrm{CC}, 7-9$ & 99.15 & 27.30 & 46.96 & 25.74 & 1.13 & Leptokurtic & 10.21 & 2.64 \\
\hline $13 X-2,15-17$ & 108.75 & 27.82 & 52.82 & 19.36 & 3.03 & Leptokurtic & 45.07 & 4.28 \\
\hline $13 \mathrm{X}-\mathrm{CC} .20-22$ & 109.52 & 28.91 & 52.85 & 18.24 & 2.33 & Leptokurtic & 40.29 & 3.83 \\
\hline $14 X-2,80-82$ & 119.00 & 34.48 & 53.33 & 12.55 & 1.37 & Leptokurtic & 19.45 & 1.95 \\
\hline $15 X-1,80-82$ & 127.10 & 34.34 & 65.66 & 0.00 & 1.03 & Leptokurtic & 12.76 & 1.32 \\
\hline $15 \mathrm{X}-\mathrm{CC}, 20-22$ & 127.66 & 32.59 & 67.41 & 0.00 & 1.02 & Leptokurtic & 12.55 & 1.46 \\
\hline $16 X-3,30-32$ & 139.24 & 34.85 & 44.99 & 20.16 & 2.14 & Leptokurtic & 37.81 & 3.56 \\
\hline $17 X-1,108-110$ & 146.68 & 34.90 & 65.10 & 8.39 & 0.84 & Leptokurtic & 8.03 & 1.50 \\
\hline $17 X-2,59-60$ & 147.69 & 42.62 & 55.84 & 1.54 & 1.06 & Leptokurtic & 8.53 & 1.69 \\
\hline $18 \mathrm{X}-\mathrm{CC}, 4-6$ & 155.24 & 32.33 & 51.24 & 16.43 & 2.47 & Leptokurtic & 27.12 & 4.66 \\
\hline $19 X-3,90-92$ & 168.80 & 31.95 & 49.91 & 18.14 & 2.38 & Leptokurtic & 43.76 & 3.96 \\
\hline $19 X-4,28-30$ & 169.37 & 26.80 & 68.16 & 5.04 & 1.12 & Leptokurtic & 19.56 & 1.49 \\
\hline $20 \mathrm{X}-1,122-124$ & 175.72 & 35.94 & 51.34 & 12.72 & 2.38 & Leptokurtic & 31.65 & 4.09 \\
\hline $22 X-1,4-6$ & 193.84 & 34.48 & 64.15 & 1.37 & 1.03 & Leptokurtic & 10.79 & 1.69 \\
\hline $22 \mathrm{X}-\mathrm{CC}, 4-6$ & 194.10 & 30.91 & 52.02 & 17.07 & 1.03 & Leptokurtic & 10.41 & 1.55 \\
\hline \multicolumn{9}{|l|}{ 160-971D- } \\
\hline IH-3, 24-26 & 3.24 & 37.66 & 57.50 & 4.37 & 2.10 & Leptokurtic & 18.00 & 5.45 \\
\hline $2 \mathrm{H}-1,19-21$ & 8.19 & 26.75 & 51.24 & 22.01 & 2.21 & Leptokurtic & 47.51 & 3.46 \\
\hline $2 \mathrm{H}-3,29-31$ & 11.29 & 39.04 & 58.51 & 2.45 & 2.08 & Leptokurtic & 13.03 & 6.61 \\
\hline $2 \mathrm{H}-4,19-2 \mathrm{I}$ & 12.69 & 39.79 & 60.21 & 0.00 & 1.02 & Leptokurtic & 9.22 & 1.62 \\
\hline $3 \mathrm{H}-1,29-31$ & 19.29 & 42.79 & 57.21 & 0.00 & 1.04 & Leptokurtic & 8.25 & 1.87 \\
\hline $3 \mathrm{H}-3,29-3 \mathrm{I}$ & 20.79 & 37.35 & 62.32 & 0.33 & 1.14 & Leptokurtic & 10.90 & 2.13 \\
\hline $3 \mathrm{H}-6,29-31$ & 25.29 & 40.92 & 57.80 & 1.28 & 1.83 & Leptokurtic & 10.63 & 7.89 \\
\hline $4 \mathrm{H}-1,110-112$ & 28.10 & 33.62 & 66.38 & 0.00 & 0.94 & Leptokurtic & 11.07 & 1.25 \\
\hline $4 \mathrm{H}-3,110-112$ & 31.10 & 35.38 & 59.26 & 5.36 & 0.98 & Leptokurtic & 9.47 & 1.53 \\
\hline $4 \mathrm{H}-5,20-22$ & 33.20 & 36.46 & 58.37 & 5.18 & 1.23 & Leptokurtic & 11.34 & 2.47 \\
\hline $5 \mathrm{H}-1,120-122$ & 37.20 & 32.85 & 62.75 & 4.40 & 1.09 & Leptokurtic & 11.41 & 1.97 \\
\hline $5 \mathrm{H}-4,20-22$ & 41.20 & 32.57 & 65.35 & 2.42 & 1.12 & Leptokurtic & 11.54 & 2.42 \\
\hline $5 \mathrm{H}-5,119-12 \mathrm{I}$ & 43.69 & 29.97 & 59.85 & 10.17 & 2.72 & Leptokurtic & 40.47 & 4.59 \\
\hline \multicolumn{9}{|l|}{ 160-971E- } \\
\hline $1 \mathrm{H}-2,14-16$ & 1.64 & 38.20 & 61.80 & 0.00 & 1.08 & Leptokurtic & 9.93 & 1.96 \\
\hline $1 \mathrm{H}-3,14-16$ & 3.14 & 35.20 & 58.85 & 5.95 & 3.15 & Leptokurtic & 28.35 & 6.12 \\
\hline IH-4.14-16 & 4.64 & 37.26 & 57.81 & 4.93 & 2.20 & Leptokurtic & 17.30 & 5.60 \\
\hline $2 \mathrm{H}-4,35-37$ & 14.35 & 32.68 & 59.95 & 4.37 & 2.27 & Leptokurtic & 16.81 & 6.48 \\
\hline $2 \mathrm{H}-7,35-37$ & 18.75 & 35.53 & 61.90 & 2.58 & 1.70 & Leptokurtic & 13.37 & 6.27 \\
\hline $3 \mathrm{H}-7,18-20$ & 27.30 & 37.85 & 59.56 & 2.59 & 2.86 & Leptokurtic & 14.67 & 11.06 \\
\hline
\end{tabular}

Note: Statistical parameters (standard deviation, kurtosis, skewness) defined in McManus (1988). 